\title{
Multiplexing and demultiplexing logic functions for computing signal processing tasks in synthetic biology
}

\author{
Lorenzo Pasotti · Mattia Quattrocelli · Daniela Galli • \\ Maria Gabriella Cusella De Angelis • Paolo Magni
}

Received: date / Accepted: date

\begin{abstract}
Building biological devices to perform computation and signal processing tasks is one of the main research issues in Synthetic Biology. Here, two modular biological systems which could mimic multiplexing and demultiplexing logic functions are proposed and discussed. These devices, called multiplexer (mux) and demultiplexer (demux) respectively, have a remarkable importance in electronic, telecommunication and signal processing systems and, similarly, they could play a crucial role if implemented in a living organism such as E. coli. BioBrick standard parts have been used to design mux an demux and to construct two genetic circuits that could carry out the desired tasks. A modular approach, mimicking basic logic gates (AND, OR and NOT) with protein/autoinducer or protein/DNA interactions and interconnecting them to create the final circuits, was adopted. A mathematical model of the designed gene networks has been defined and simulations have been performed to validate the expected behaviour of the systems. In addition, circuit subparts have been tested in vivo and results have been used to determine some of the parameters of the mathematical model. According to both the experimental and simulated results, guidelines for future finalization of mux and demux are provided.
\end{abstract}

Keywords Multiplexing · In vivo signal processing · Logic gates · BioBricks · Quorum sensing · PoPS ·

Mathematical modeling of gene networks

L. Pasotti · P. Magni

Dipartimento di Informatica e Sistemistica, Università degli Studi di Pavia, via Ferrata 1, I-27100, Pavia, Italy

E-mail: lorenzo.pasotti@unipv.it, paolo.magni@unipv.it

M. Quattrocelli · D. Galli · M.G. Cusella De Angelis

Centro di Ingegneria Tissutale, Università degli Studi di Pavia, via Ferrata 1, I-27100, Pavia, Italy

E-mail: mattiaq@hotmail.it,dgalli05@yahoo.it,cusella@unipv.it 


\section{Introduction}

Signal processing is an essential task in electronic engineering systems and for much of modern technology in almost all areas of life. Even in naturally occurring biological systems enormous amounts of information are processed to perform coordinated complex tasks [1]. An important class of electronic circuits is made of digital ones. They are designed interconnecting basic building blocks, called logic gates, following specific rules to obtain the desired functions. A logic gate is a computing unit that performs a logical operation on one or more logic inputs and produces a single output. Gates are identified by their function and can be placed in parallel or serial combinations in order to perform more complex functions.

The architecture of the natural gene regulatory networks is reminiscent of electronic logical circuits. However, while for digital logic inputs are either on or off, biological logic sometimes leads to intermediate induction levels [2]. Nevertheless, due to their sigmoid-shaped response curves, gene regulation systems can be considered genetic analog-digital converters. The signal is either on or off for a wide range of input concentrations, with the system changing between the on and off states in a relatively small concentration window [3].

For these reasons, one of the fields explored by the Synthetic Biology researchers has been the engineering of microorganisms to implement logical functions in biological chassis [4, 5, 6, 7, 8, 9]. CAMBIATO Synthetic Biology applies many of the principles of engineering to the field of Biology in order to create biological devices which can ultimately be integrated into increasingly complex systems. These principles include standardization of parts, modularity, abstraction, reliability, predictability, and uniformity. The application of engineering principles to biology is complicated by the inability to predict the functions of even simple devices and modules, within the cellular environment. Some of the confounding factors are gene expression noise, mutation, cell death, undefined and changing extracellular environments, and interactions with the cellular context [10]. In Synthetic Biology, logic gates are created by engineering the biochemical reactions that regulate various cellular processes, such as transcription, translation, protein phosphorylation, allosteric regulation, ligand/receptor binding, and enzymatic reactions. Although the diversity of biochemical reactions can make it difficult to combine different devices, these logic gates can be used to build complex systems with functions that have many practical applications [11].

\section{NUOVO}

Synthetic Biology aims to apply the main concepts of the engineering field, such as modularity, standardization and abstraction, to the biological sciences world to produce devices which can be connected together and ultimately be incorporated into systems of higher complexity. The incapability of predicting the function of relatively 
simple parts and devices complicates the effective application of these concepts. DNA mutations, noise in gene expression, metabolic burden, crosstalk among devices and cellular context and undefined changes in the extracellular environments are some of the confounding factors [10]. The construction of logic gates in Synthetic Biology is performed by engineering the biological and biochemical interactions involved in different cellular processes at different levels. Even if the diversity of reactions, such as transcription, post-transcriptional modifications, translation, enzyme reactions, protein phosphorylation or binding with specific molecules, can complicate the interconnection of logic gates, it is possible to use these modules to construct complex composite devices which may be applied to various fields [11].

So, biological implementation of logic gates is definitely a powerful resource to design modular gene networks inspired to digital electronic circuits $[3,6]$. Highly important functions in synthetic biological systems include the processing, the manipulation and the computation of exogenous or endogenous signals, which can be effectively useful to engineer novel complex behaviours [12].

In this work, these concept have been used to design synthetic biological systems that could carry out multiplexing and demultiplexing functions.

\subsection{Multiplexer and demultiplexer}

Multiplexing is a function where one of multiple input signals is conveyed into a single output channel, whereas in demultiplexing a single input signal is conveyed into one of multiple output channels. The choice of the input channel in multiplexing and of the output channel in demultiplexing is controlled by a selector. The devices implementing these two functions are called multiplexer (mux) and demultiplexer (demux) respectively and can be considered as controlled switches [13]. They have a remarkable importance in electronic, telecommunication and signal processing systems, like central processing units or analog to digital converters.

In this work, only two-input mux (mux 2:1) and two-output demux (demux 1:2) were considered. Furthermore, only digital on/off signals, i.e. they can only assume Boolean values (0 or 1), were considered. In this framework, mux and demux can be considered as logic functions and, using truth tables and Karnaugh maps methods, it is possible to design a modular logic network for each of the two devices [14].

In particular, the overall behaviour of mux and demux is summarized in Fig. 1. According to their logic functions, the basic logic gates composing the networks are AND, OR, NOT for mux and AND, NOT for demux. 
A

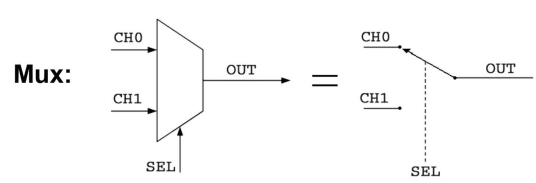

Demux:

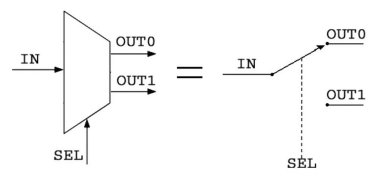

B
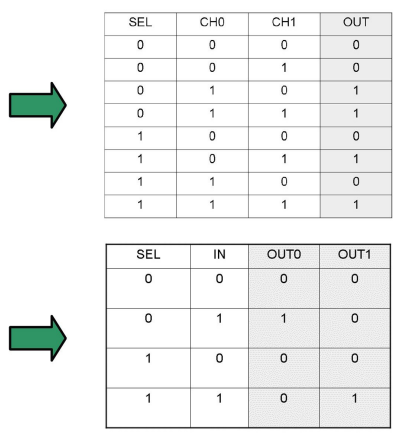

$\mathrm{C}$
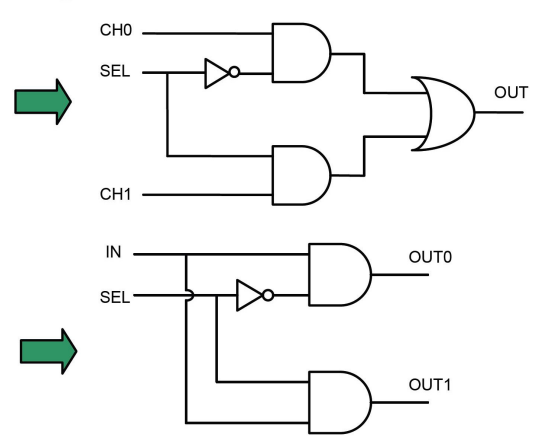

Fig. 1 Mux and demux schematic structures (A), truth tables (B) and logic networks obtained by Karnaugh maps methods (C). $\mathrm{CH} 0$ and $\mathrm{CH} 1$ are the two input channels of mux, OUT the output channel of the demux; IN is the input channel of demux, OUT0 and OUT1 the two output channels of demux; SEL is to selector. In the truth tables, 1 and 0 represent on and off signals, respectively.

The goal of this work is to design genetic networks that could mimic mux and demux in a biological chassis, such as E. coli, using standard BioBrick parts available in the Registry of Standard Biological Parts (http://partsregistry.org) and to discuss the working conditions of these devices, also thanks to the definition and the implementation of suitable mathematical models. Protein/DNA and autoinducer/protein interactions were used to build up the required biological logic gates.

\subsection{Design of biological gates}

Fig. 2 summarizes from both a digital electronics and a biological point of view the proposed AND (A), OR (B) and NOT (C) gates. In particular, AND gate has been implemented using quorum sensing complexes, such as lux operon from Vibrio fischeri [15] and las operon from Pseudomonas aeruginosa [16]. In lux system, Plux right promoter (BBa_R0062 ${ }^{1}$ ) can be activated only by the simultaneous expression of luxR (BBa_ C0062) and luxI (BBa_C0061) genes, which encode the LuxR transcription activator and the synthase of the autoinducer N-3-oxohexanoyl-L-homoserine lactone (3OC6HSL), required to activate LuxR, respectively [17, 18, 19, 20]. Las system shows a similar behaviour: it is composed by Plas promoter (BBa_R0079) and lasR (BBa_C0079) and lasI (BBa_C0078) genes [21, 22, 23], which work through N-3-oxododecanoyl-L-homoserine lactone (3OC12HSL) autoinducer and can implement an AND gate as well [9].

\footnotetext{
1 BBa_code is the official reference number of the BioBrick in the Registry of Standard Biological Parts.
} 
A biological OR gate has been implemented simply replicating a gene of interest under the control of two independently inducible promoters, Plux and Plas.

A biological NOT gate has been implemented using lambda-cI system from lambda phage [24]. This system includes Plambda repressible promoter (BBa_R0051), which is normally on, but can be turned off upon the expression of $\mathrm{cI}$ gene (BBa_C0051), which encodes a specific repressor for this promoter [25].

A

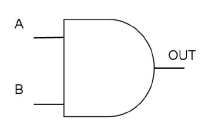

\begin{tabular}{|c|c|c|}
\hline $\mathrm{A}$ & $\mathrm{B}$ & OUT \\
\hline 0 & 0 & 0 \\
\hline 0 & 1 & 0 \\
\hline 1 & 0 & 0 \\
\hline 1 & 1 & 1 \\
\hline
\end{tabular}

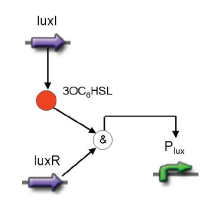

\begin{tabular}{|c|c|c|}
\hline lasl & lasR & $\mathrm{P}_{\text {las }}$ \\
\hline luxl & luxR & $\mathrm{P}_{\text {lux }}$ \\
\hline no & no & off \\
\hline no & yes & off \\
\hline yes & no & off \\
\hline yes & yes & on \\
\hline
\end{tabular}

B

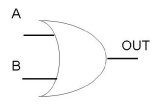

\begin{tabular}{|c|c|c|}
\hline $\mathrm{A}$ & $\mathrm{B}$ & OUT \\
\hline 0 & 0 & 0 \\
\hline 0 & 1 & 1 \\
\hline 1 & 0 & 1 \\
\hline 1 & 1 & 1 \\
\hline
\end{tabular}

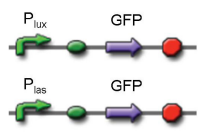

$\mathrm{C}$

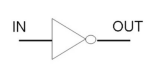

\begin{tabular}{|c|c|}
\hline IN & OUT \\
\hline 0 & 1 \\
\hline 1 & 0 \\
\hline
\end{tabular}

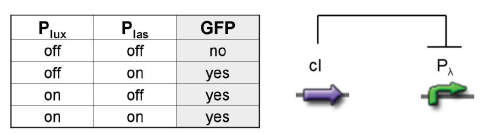

\begin{tabular}{|c|c|}
\hline cl & $\mathbf{P}_{\boldsymbol{\lambda}}$ \\
\hline no & on \\
\hline yes & off \\
\hline
\end{tabular}

Fig. 2 Logic gates design: AND gate was implemented with the lux (or las) system, where only the simultaneous presence of LuxI (or LasI) and LuxR (or LasR) can activate Plux (or Plas) (A); OR gate was implemented by replicating the gene encoding the function of interest downstream of two independent promoters (B); NOT gate was implemented through the cI-Plambda repressible system (C). Graphic symbols: curved arrows represent promoters, straight arrows represent coding sequences, ovals represent RBSs, octagons represent transcriptional terminators and circles represent autoinducers.

\subsection{Design of biological MUX and DEMUX}

The interconnection of the described biological logic gates, according to the logic networks shown in Fig. 1, gives life to the final circuits that could theoretically yield mux and demux behaviour. In order to supply two universal devices that can detect any kind of input signal and that can express any protein as output, mux and demux circuits have been designed without inputs and outputs; in this way, final users can specialize the circuits assembling the specific input and output devices for the desired applications, following the guidelines provided in this paper.

Fig. 3 shows the resulting circuits and all the possible interactions between the networks elements. In this figure, generic input (PA, PB, PS for mux, PI and PS for demux) and output (GOI for mux, GOI0 and GOI1 for demux) elements are present. In particular, PA, PB, PS and PI are promoters that can be activated respectively by the generic molecules A, B, S and I, while GOI, GOI0 and GOI1 are the genes of interest, which encode for the 
generic output proteins GOI, GOI0 and GOI1. According to these genetic circuits, if A, B, S and GOI correspond respectively to IN0, IN1, SEL and OUT for mux and if I, S, GOI0 and GOI1 correspond respectively to IN, SEL, OUT0 and OUT1 for demux, the biological truth tables of mux and demux are analogous to the Boolean truth tables of Fig. 1.

A

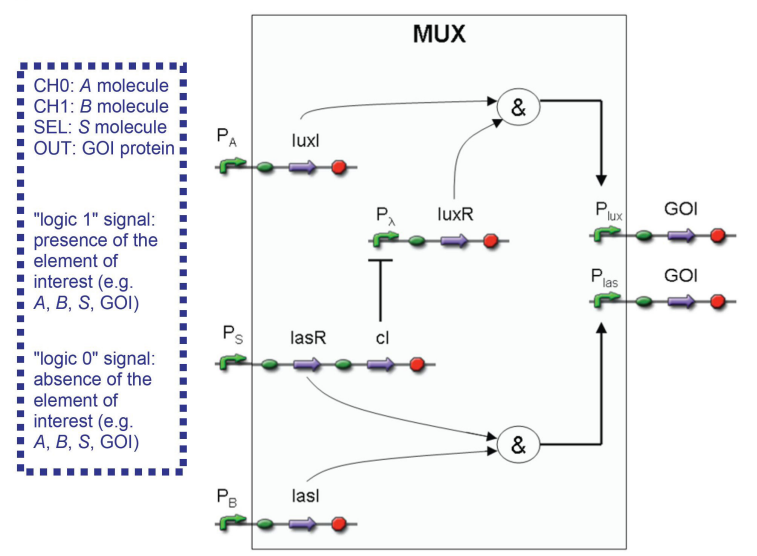

B

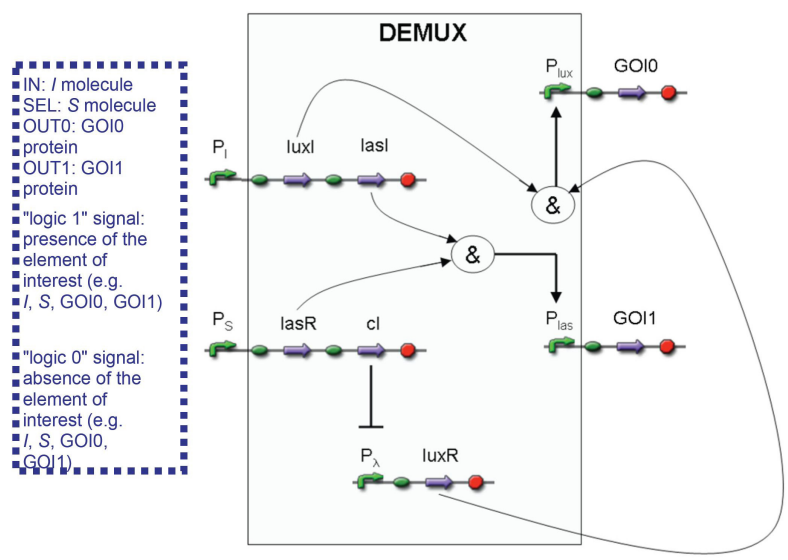

Fig. 3 Biological mux (A) and demux (B) circuits. PA, PB, PS and PI indicate promoters activated respectively by the generic molecules A, B, S and I. GOI, GOI0 and GOI1 indicare the genes of interest. Graphic symbols: curved arrows represent promoters, straight arrows represent coding sequences, ovals represent RBSs, octagons represent transcriptional terminators, \& indicates that two elements are required to activate a promoter.

Three final biological devices for mux and two for demux (see Fig. 4A) have been planned in order to build up the designed circuits. Each planned device has a ribosome binding site (RBS) at 5' end and a promoter or a transcriptional terminator at 3' end. For this reason, mux and demux circuits both conform to Polymerase Per Second (PoPS) device boundary standard in a multi-input multi-output framework [26].

In this paper, mathematical modeling has been used to study the theoretical behaviour of the designed networks and experiments have been performed on a set of constructed genetic sub-circuits, using GFP (Green Fluorescent Protein) or RFP (Red Fluorescent Protein) as reporters. These sub-circuits are essential parts of both mux and demux and, when interconnected and placed together with the other modules, they may contribute to yield the theoretically behaviour of mux and demux. 


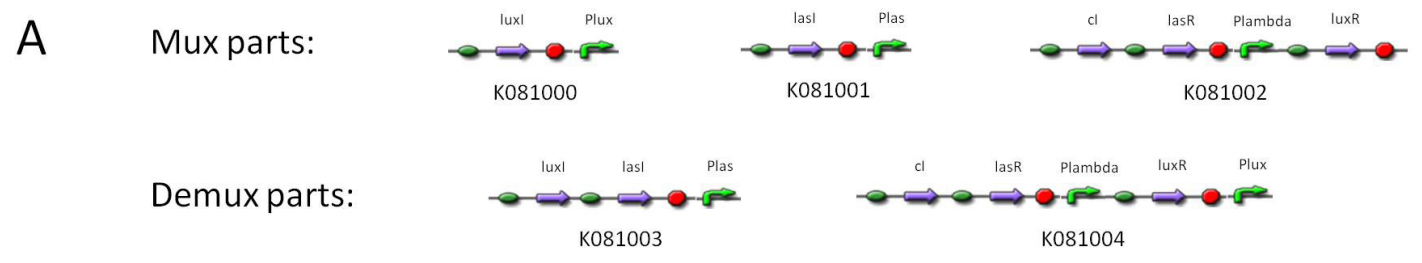

B Intermediate parts submitted to the Registry:

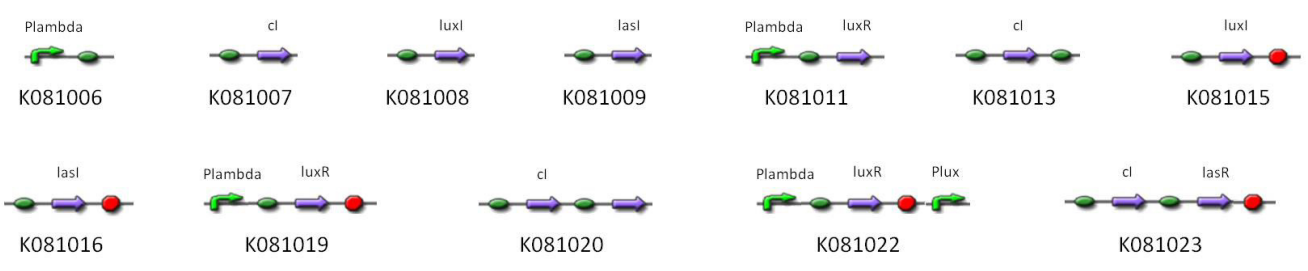

\section{Test parts:}
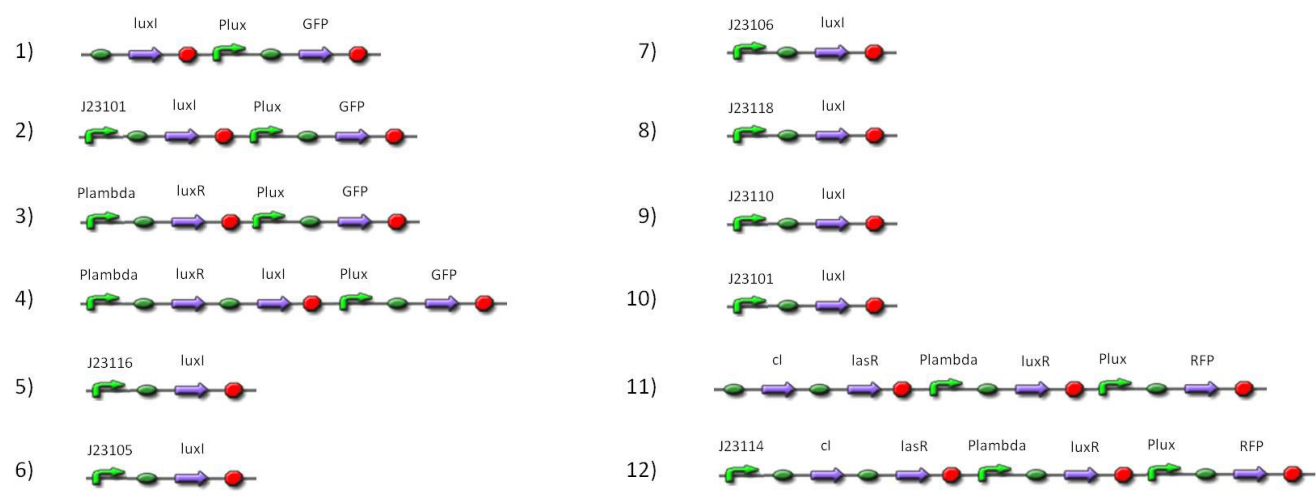

Fig. 4 Final devices for mux and demux (A). Intermediate parts submitted to the Registry (B). Test parts used for subcircuit validation (C). The reporter genes are GFPmut3b (BBa_E0040) or mRFP1 (BBa_E1010). All the ribosome binding sites are $\mathrm{BBa} \_\mathrm{B} 0030$ and all the transcriptional terminators are BBa_B1006, except for test parts \#5-10 in which the terminator is BBa_B0015. J231xx are constitutive promoters. Graphic symbols: curved arrows represent promoters, straight arrows represent coding sequences, ovals represent RBSs, octagons represent transcriptional terminators.

\section{Materials and Methods}

2.1 Cloning and parts construction

BioBrick parts from the MIT Registry of Standard Biological Parts (http://partsregistry.org) were used to construct all the plasmids. They were assembled according to the BioBrick Standard Assembly [27]. In particular, parts from MIT Spring 2008 DNA Distribution (http://2008.igem.org), spotted on filter paper, were resuspended in warm $\left(55^{\circ} \mathrm{C}\right) \mathrm{TE}$ buffer 10:1, pH 8.0 and used to transform TOP10 competent E. coli (Invitrogen) by heat-shock, 
according to the manufacturer protocol. Plasmids were purified through QIAprep Spin Miniprep kit (Qiagen) from $5 \mathrm{ml}$ overnight cultures. For every plasmid, $800 \mu \mathrm{l}$ of culture were mixed with $200 \mu \mathrm{l}$ of sterile glycerol for long-term storage at $-80^{\circ} \mathrm{C}$. Parts or vector bulks were obtained through EcoRI, XbaI, SpeI, PstI (Roche) digestion of $1 \mu \mathrm{g}$ of DNA for $3 \mathrm{~h}$ at $37^{\circ} \mathrm{C}$ and isolated from $1 \%$ agarose gel by DNA Gel Extraction Kit (Roche). Cloning of parts was assessed by T4 Ligase (Roche) activity on XbaI-PstI inserts downstream of SpeI-PstI parts or EcoRI-SpeI inserts upstream of EcoRI-XbaI parts. Ligations were performed overnight at $16^{\circ} \mathrm{C}$ in $10 \mu \mathrm{l}$ with a molar ratio 1:6 (bulk:insert) and heated at $65^{\circ} \mathrm{C}$ for $20^{\prime}$ before proceeding to heat-shock transformation of TOP10.

\subsection{Fluorescence assays}

1-5 $\mu \mathrm{l}$ of recombinant bacteria were recovered from thawed glycerol stocks and were grown in 1-5 ml of selective M9 supplemented medium (M9 salts, $1 \mathrm{mM}$ thiamine hydrochloride, $0.2 \%$ casamino acids, $2 \mathrm{mM} \mathrm{MgSO} 4,0.1 \mathrm{mM}$ $\mathrm{CaCl} 2,0.4 \%$ glycerol, ampicillin $100 \mu \mathrm{g} / \mathrm{ml}$ ) for about 20 hours at $37^{\circ} \mathrm{C}$ and $220 \mathrm{rpm}$ shaking.

Cultures were diluted 1:100 in 1-5 $\mathrm{ml}$ of fresh selective M9 and incubated for 5 hours under the same conditions as before. $200 \mu \mathrm{l}$ aliquots were transferred into a flat-bottom 96-well microplate (Greiner) and assayed for 3 hours with an automatically repeating protocol with the Infinite F200 microplate reader (Tecan): fluorescence (Ex.485 nm, Em.540 nm, gain=50 for GFP and Ex.535 nm, Em.620 nm, gain=50 for RFP) and absorbance $(600 \mathrm{~nm})$ were measured every 5 minutes. Temperature was kept constant at $37^{\circ} \mathrm{C}$ and every 5 minutes cultures were shaken $(3$ mm amplitude, linear shaking) for $15 \mathrm{sec}$.

Induction experiments were performed analogously, except that cultures were induced in the microplate wells with $2 \mu \mathrm{l}$ of 3OC6HSL (Sigma Aldrich \#K3007) to yield the desired final concentration.

The absorbance of $200 \mu \mathrm{l}$ of sterile M9 and the autofluorescence of a TOP10 culture without GFP and RFP were measured in order to estimate the absorbance and fluorescence background, respectively.

M9 and TOP10 background values were subtracted to all the absorbance and fluorescence raw measurements to obtain values proportional to the per-well cell count and number of fluorescence molecules. The fluorescent reporter protein synthesis rate per cell time series of each culture was computed as the numeric time derivative of the fluorescence values, divided by absorbance. This time series was averaged over a 2-hour period during the bacterial exponential growth phase and, when dealing with induced cultures, after about 50 minutes from the induction time [26]. 
2.3 3OC6HSL production assay

Bacteria bearing the RBS-luxI test parts were recovered from thawed glycerol stocks and were grown in 1-5 ml of selective M9 supplemented medium for about 20 hours at $37^{\circ} \mathrm{C}$ and $220 \mathrm{rpm}$ shaking.

Cultures were diluted 1:100 in 1-5 ml of fresh selective M9 and incubated for 8 hours under the same conditions as before. Cultures were centrifuged $(7000 \mathrm{rpm}, 10 \mathrm{~min})$ and the supernatant was filtered $(0.2 \mu \mathrm{m})$ to remove all the bacterial cells. Supernatants were stored at $-20^{\circ} \mathrm{C}$ until further use.

TOP10 bearing BioBrick BBa_T9002 in pSB1A3 high copy vector were used as a 3OC6HSL biosensor which synthesizes GFP as a function of 3OC6HSL concentration [26]. TOP10 with BBa_T9002 were recovered from thawed glycerol stocks and were grown in 1-5 $\mathrm{ml}$ of M9 supplemented medium for about 20 hours. The culture was diluted 1:100 in a proper volume of fresh selective M9 and, after 5 hours, $180 \mu$ l aliquots were transferred in a 96-well microplate. Wells were induced with $20 \mu \mathrm{l}$ of the supernatants obtained before and the BBa_T9002 culture was assayed as described above in the microplate reader for 3 hours. Standard calibration curves were obtained by inducing the wells with $20 \mu \mathrm{l}$ of M9 supplemented with 3OC6HSL at different autoinducer concentrations. When the measured concentrations were higher than the biosensor upper detection limit, the supernatants were diluted properly with fresh M9 in a final volume of $20 \mu \mathrm{l}$, which was used to induce BBa_T9002 wells. Measurements were performed in triplicate. Background values for absorbance and fluorescence were subtracted as described above. The average GFP synthesis rate per cell was computed over a 3-hour period to indirectly measure the 3OC6HSL concentration.

2.4 Mathematical model

Ordinary differential equations with Hill functions were used to model the mux and demux genetic circuits. Equations and model parameters definition are reported in the Supplementary File 1, as well as the parameter estimation procedures performed on experimental data expressly acquired as a part of this study. Model was implemented in MATLAB (MathWorks, Natick, MA) routines and all the simulations reported in this paper were performed by using MATLAB 2010a suite. Note that RFP was considered as the mux single output (GOI), while RFP and GFP were considered as the two outputs of demux (GOI0 and GOI1, respectively). 


\section{Results}

3.1 Physical assemblies

All the five planned final devices which could lead to mux and demux functions are shown in Fig. 4A and have been built up and submitted to the Registry of Standard Biological Parts, as well as an exhaustive documentation which includes user's assembly handbooks and gene networks schemes and description. BBa_K081000 and BBa_K081001 devices are contained in pSB1A2 high copy number plasmid (pUC19-derived pMB1 replication origin) carrying Ampicillin resistance, while BBa_K081002, BBa_K081003 and BBa_K081004 devices are contained in pSB1AK3 high copy number plasmid (same replication origin as pSB1A2) carrying Ampicillin and Kanamycin resistance. Intermediate parts (shown in Fig. 4B) have been shared and documented in the Registry as well. In order to validate the behaviour of the implemented circuits, additional composite test parts have been built up (see Fig. 4C).

\subsection{Parts characterization}

The AND gate constructed with the lux system was quantitatively characterized. TOP10 bearing test parts \#1-4 allowed to validate the four rows of the AND gate reported in Fig. 2A. This logic gate is expected to give a high output only when both luxI and luxR genes are expressed. Results show that, in this condition, the output is about 100-fold higher than the low output values (Fig. 5).

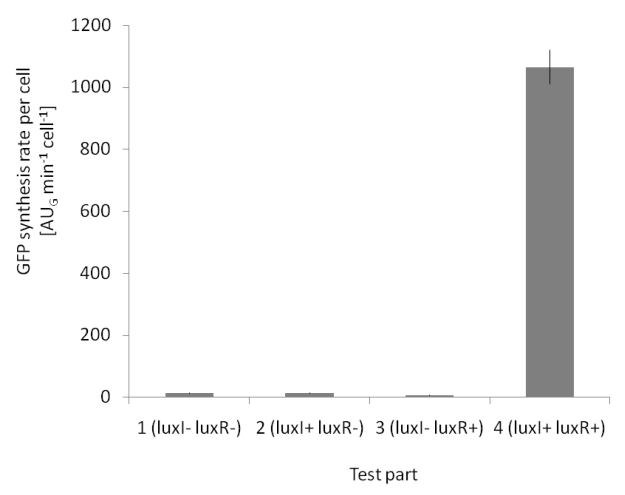

Fig. 5 Results of the AND gate designed with the lux system. Error bars represent the standard deviation of the measurements of three assayed clones. $A U_{G}$ indicates arbitrary units of GFP. 
Test part \#3 was also used to evaluate the behaviour of Plux in presence of different concentrations of exogenous 3OC6HSL, when luxR is constitutively expressed. The "static" characteristic is reported in Fig. 6 as a function of $3 \mathrm{OC} 6 \mathrm{HSL}$ and this result is consistent with previous reports [26]. Moreover, it is important to note that the maximal GFP output for test part \#3 is the same as test part \#4, thus demonstrating the full induction of this system by exogenously adding 3OC6HSL or by endogenously producing 3OC6HSL via luxI (compare Fig. 5 and Fig. 6).

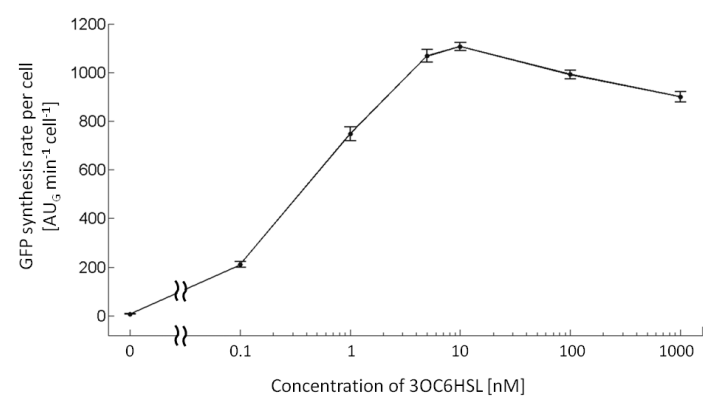

Fig. 6 Test part \#3 behaviour in presence of exogenous 3OC6HSL. Error bars represent the standard deviation of the measurements of three assayed clones. $A U_{G}$ indicates arbitrary units of GFP.

Test parts \#5-10 were used to characterize the capability of RBS-luxI to produce 3OC6HSL when induced at different expression strengths. This information is essential when dealing with mux CH0 and demux input because RBS-luxI is directly interfaced with an input promoter in both devices. These test parts are constitutive generators of luxI, driven by promoters of different strength. The constitutive promoters BBa_J23101, BBa_J23105, BBa_J23106, BBa_J23110, BBa_J23116 and BBa_J23118, were ranked via RFP measurement (Fig. 7A). These promoters, taken from the Registry, were contained in BBa_J61002 high copy vector, which carries RBS-RFPTerminator downstream of the SpeI restriction site, adjacent to the promoter. For this reason, they were ready to be measured without performing any assembly.

3OC6HSL was measured in the supernatant of cultures bearing test parts \#5-10 (Fig. 7B) through the BBa_T9002 biosensor, whose estimated detection limit was $0.1 \mathrm{nM}$ (data not shown). The concentration of 3OC6HSL in the growth medium of these cultures was correlated to the upstream promoter strength, giving high concentrations of autoinducer when stronger promoters produce luxI and low concentrations in case of weaker promoters (Fig. 7C), following a nonlinear trend. 

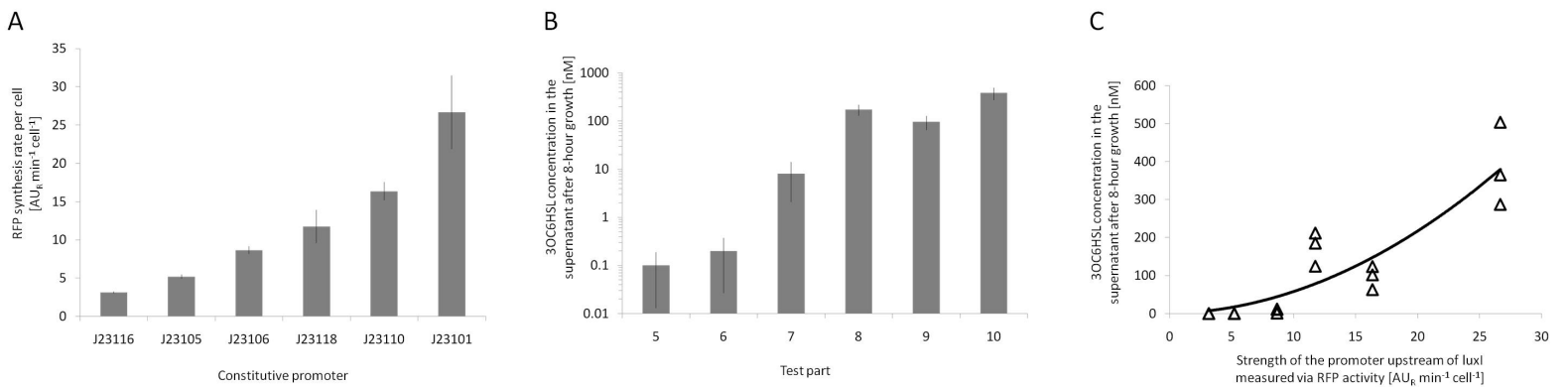

Fig. 7 Characterization of the luxI protein generators (test parts \#5-10). A) Constitutive promoters ranking via RFP measurement. Error bars represent the standard deviation of the measurements of three assayed clones. B) 3OC6HSL production after an 8-hour growth. Error bars represent the standard deviation of the measurements of three independent experiments. C) 3OC6HSL concentration as a function of promoter strength (diamonds) with a second degree polynomial fit (solid line, $\left.\mathrm{R}^{2}>0.8\right)$. $A U_{R}$ indicates arbitrary units of RFP.

The NOT gate was also characterized when coupled to the previously studied luxR-Plux system, which is a fundamental part of the AND gate, through test parts \#11-12 via RFP measurement. The genetic circuit of test part \#11 is expected to express luxR through the Plambda promoter, which is not repressed because cI is not produced. So, in presence of 3OC6HSL the Plux promoter can be turned on. When a promoter is placed upstream of this circuit, thus yielding test part \#12, cI is produced and it can repress Plambda. In this case, luxR cannot be produced and Plux cannot be turned on upon 3OC6HSL exogenous addition. Induction results for test part \#12 show that, when the expression of cI is driven by BBa_J23114 promoter, RFP activity is lower than in test part \#11 for inducer concentrations up to $100 \mathrm{nM}$ of 3OC6HSL (Fig. 8). This difference is probably due to the partial repression of Plambda promoter in test part \#12, so luxR expression is reduced when compared to test part \#11 and the activation of Plux promoter is weaker, as expected. However, this difference cannot be appreciated for 1-10 $\mu \mathrm{M}$ inductions. As illustrated below, mathematical model predictions are in accordance with this experimental result and then the right working conditions of the NOT gate will be discussed later. It is important to note that BBa_J23114 activity is about 33\% of BBa_J23116, the weaker promoter of Fig. 7A (data not shown). This suggests that the NOT gate is highly sensitive to the transcriptional input and even a low transcription rate can repress Plambda promoter.

\subsection{Mathematical model simulations}

Input $\left(P_{A}, P_{B}\right.$ for mux and $P_{I}$ for demux) and selector $\left(P_{S}\right)$ promoter values were varied to span all the truth table combinations of both devices. Fluorescence output values, in terms of RFP (for mux) or RFP and GFP (for 


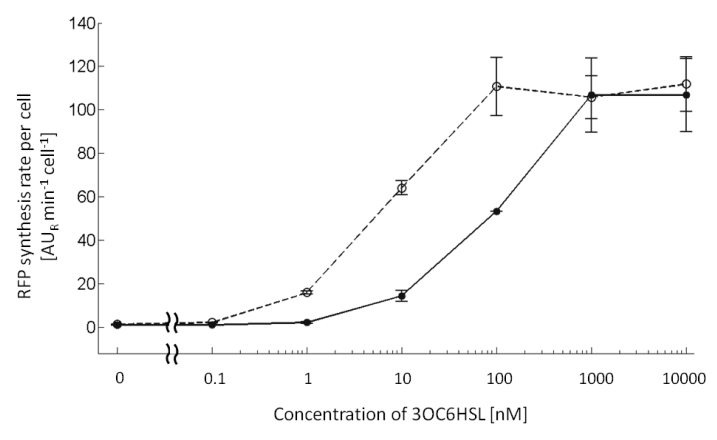

Fig. 8 Results of the NOT gate: Plux activity as a function of 3OC6HSL for test part \#11 (dashed line and open circles) and \#12 (continuous line and filled circles). Error bars represent the standard deviation of the measurements of three assayed clones. $A U_{R}$ indicates arbitrary units of RFP.

demux) synthesis rate per cell, were predicted after 16 hours starting from an inoculum of $3.6 * 10^{7}$ cells. In all the simulations, initial conditions were set to zero for all the species, except LuxR which was supposed to be already present in cells at the steady-state level $\left(\alpha_{\lambda} / \gamma_{X}\right.$, see equation 7 of the Supplementary File 1). Fig. 9A shows the simulated results for mux and demux as a function of the input/selector promoters activity in different on or off conditions.

When on state was $3 A U_{R}$ min $^{-1}$ cell $^{-1}$ (same strength of BBa_J23116) and off state was 0.03 (i.e. $1 \%$ of the on state), mux and demux output values showed the expected behaviour with 5 - and 11-fold change ${ }^{2}$ between the on and off states respectively (Fig. 9A, case \#1). However, amongst the on state output values of mux, RFP could vary up to 5.5 -fold. This behaviour is due to the OR gate architecture, in which RFP is produced either by the strong promoter Plux or by the weaker promoter Plas.

In order to simulate the systems in a less ideal condition, off state activity was increased to 0.3 (i.e. $10 \%$ of the on state) for all the promoters. The difference between on and off states was lower when compared to the previous case, in fact it was 1.7- and 1.9-fold for mux and demux, respectively (Fig. 9A, case \#2). Nevertheless, both systems were still functional.

If input promoters activity in the on state was increased from 3 to $26.6 \mathrm{AU}_{R} \mathrm{~min}^{-1} \mathrm{cell}^{-1}$ (same strength of BBa_J23101), with the selector activity as in case \#1, both systems lost the expected behaviour (Fig. 9A, case \#3). In fact, a threshold between on and off states cannot be defined in both mux and demux. On the other hand,

\footnotetext{
${ }^{2}$ Fold-change was computed as the ratio between the minimum value of the on state and the maximum value of the off
} state. 
A

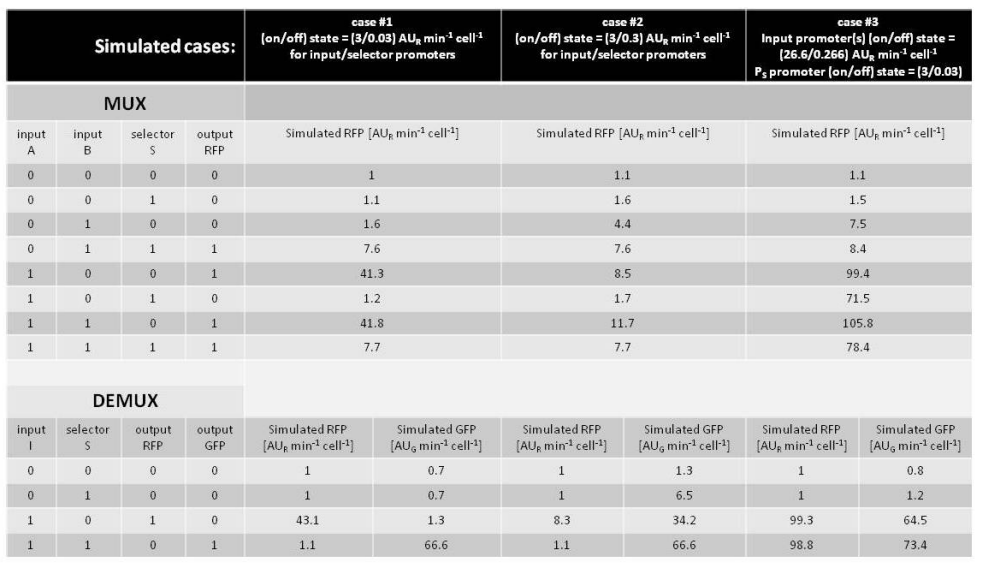

B

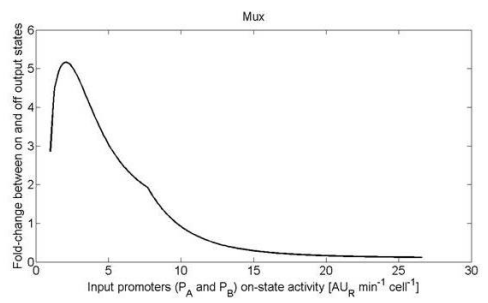

Demex

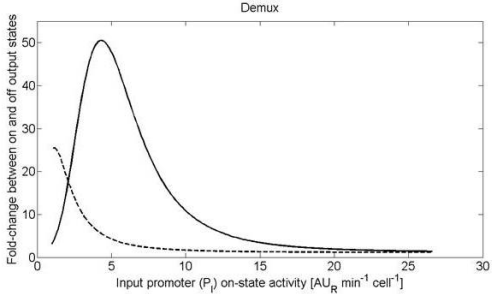

D
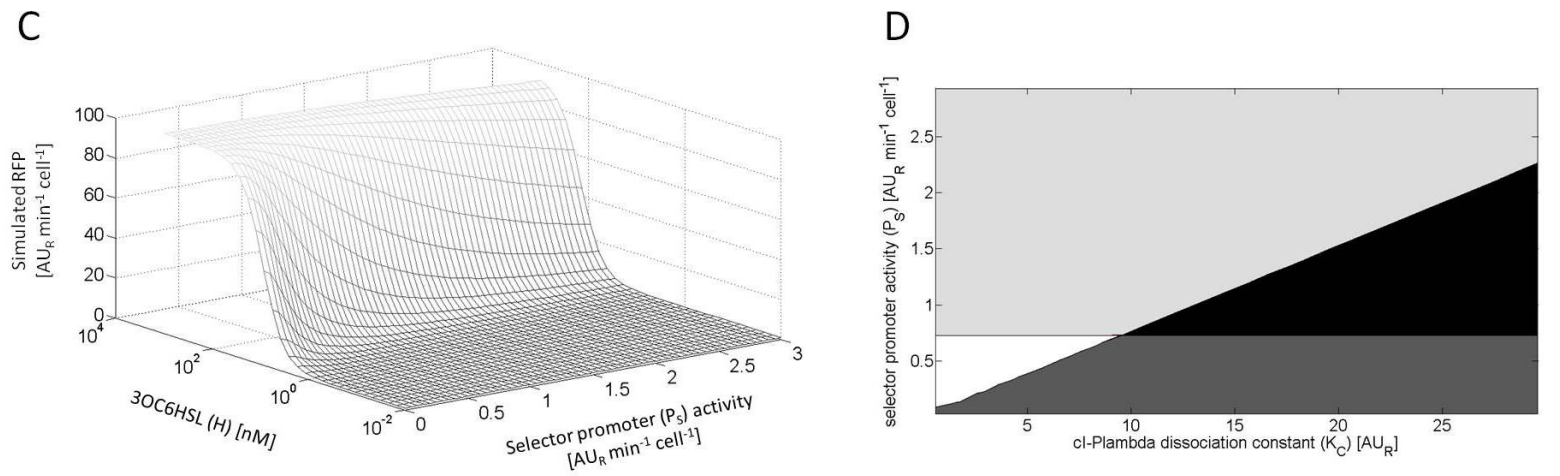

Fig. 9 A) Simulated output values of mux and demux in three conditions (the reported values for the two demux output channels correspond to RFP and GFP activities and are expressed in different arbitrary units); B) Fold-change difference between on and off output states in mux and demux as a function of input promoters $\left(P_{A}\right.$ and $P_{B}$ for mux, $P_{I}$ for demux $)$ on state activity. $P_{S}$ on state activity was set at $3 A U_{R} \mathrm{~min}^{-1} \mathrm{cell}^{-1}$; the off state activity was set at $1 \%$ of the on activity for all the promoters. In demux graph, continuous line is referred to RFP, while dashed line is referred to GFP; C) model prediction of RFP for the NOT gate coupled to the luxR-Plux system (see test part \#11) as a function of 3OC6HSL (H) and the activity of the promoter upstream of cI $\left(P_{S}\right)$; D) Simulated output of demux as a function of $P_{S}$ and $K_{C}$ referring to case \#2, panel A. On/off threshold values for RFP and GFP have been set to $4.5 A U_{R} \mathrm{~min}^{-1} \mathrm{cell}^{-1}$ and $50 \mathrm{AU} \mathrm{U}_{\mathrm{min}}^{-1}$ cell $^{-1}$, respectively. Dark and light grey zones indicate, respectively, the region in which only RFP or GFP is on. Black and white zones indicate, respectively, the region in which both or none of them are on. $A U_{R}$ and $A U_{G}$ indicate arbitrary units of RFP and GFP, respectively.

when $P_{S}$ on activity was increased to 26.6 (with an off activity of $1 \%$, keeping input promoters on and off states at 3 and 0.03 , respectively), both devices work correctly (simulation not shown). Fold-change between on and off output states was also studied as a function of the input activities in order to identify the working conditions of both devices. Results are reported in Fig. 9B. 
Moreover, the mathematical model was used to study the behaviour of the sub-system composed by the NOT gate coupled to the luxR-Plux system, described above, to better understand the causes of the unexpected experimental results obtained with test parts \#11-12 (Fig. 8). Fig. 9C reports the predicted RFP as a function of $3 \mathrm{OC} 6 \mathrm{HSL}$ and $P_{S}$ (i.e. the promoter upstream of cI). Model predictions are consistent with the experimental data described in previous section (Fig. 8). In fact, RFP is an increasing function of 3OC6HSL and it is repressed by $P_{S}$. However, when 3OC6HSL increases, the difference between RFP values in repressed (high $P_{S}$ value) and unrepressed (low $P_{S}$ value) states decreases, thus impairing the NOT gate functioning. High 3OC6HSL concentration values are related to the strong activity of $P_{A}$ and $P_{I}$ promoters. This can explain why high input promoter values are detrimental for the correct behaviour of both mux and demux, as illustrated in the simulation results above. If the LuxR maximum synthesis rate is decreased, simulations showed that RFP can be more strongly repressed at high 3OC6HSL concentrations and the NOT gate exhibits a better performance also in presence of strong $P_{A}, P_{I}$ promoters (see the Supplementary File 2).

Sensitivity analysis was carried out on cI-Plambda dissociation constant $\left(K_{C}\right)$, which showed to be critical during simulation trials. Fig. 9D reports the predicted GFP and RFP activities in demux as a function of $K_{C}$ and $P_{S}$, when input promoter $P_{I}$ is on $\left(3 A U_{R} \min ^{-1} c e l l^{-1}\right)$. This figure shows the $K_{C}$ and $P_{S}$ ranges leading to output states in which both channels are on or off (black and white regions). The same unwanted phenomena also affect the selection capability in mux (simulation not shown), in which the two input channels would not be conveyed to output in a mutually exclusive fashion.

Finally, the effect of crosstalk between lux and las systems was investigated. Results depicted that orthogonality between the two quorum sensing systems is a critical requirement for mux and demux, as even low-entity crosstalk parameter values can be detrimental. Simulation results are reported in the Supplementary File 3. 


\section{Discussion}

Mux and demux are two fundamental devices in electronics. They are used in several applications, for example in communication devices, in arithmetic logic units and, in general, in applications that involve channel sharing. Analogously, they could play a crucial role in the building of complex genetic circuits, in fact both of them can be used as controlled genetic switches [5].

Two gene networks which could lead to mux and demux logic functions were designed, built up and are available in the open source Registry of Standard Biological Parts. Their behaviour was characterized by performing simulations through an ad-hoc mathematical model, defined also thanks to in vivo experiments expressly performed as a part of this study.

Because the designed mux and demux conform to PoPS device boundary standard [26], they can be considered as general black box (PoPS in - PoPS out) devices, which can be specialized by users to detect any kind of input signal and to express any protein as output by assembling the desired input and output devices, which must conform to PoPS functional standard too. For these reasons, their application field is very wide. A biological mux can be used as a module for environmental signals, which can be selected and carried in output by modulating an exogenous selection signal. In this way, different sensing devices can be integrated in only one circuit and a Boolean logic-based programmable biosensor can be achieved. On the other hand, a biological demux can be used as a regulator for industrial or biological processes in which the sequential action of different enzymes is needed to catalyze the production of a specific molecule; in this case, an exogenous selection signal can be used to switch the synthesis of the enzymes through suitable actuator modules and thus the process can be regulated. The selection signal can be manually provided by an operator, or automatically provided by a closed-loop working system. The selection signal can even be endogenous, in fact it could be the PoPS output of a sensing device integrated in the same organism, which "observes" an internal variable of the process and controls the enzyme synthesis as a function of this variable.

Mathematical model simulation results showed that both circuits can behave as expected, but only under specific conditions. In fact, thanks to the availability of a mathematical model, it was possible to highlight some crucial points that should be considered as guidelines for future work. First, in order to have mux and demux that work as expected, their input and selector promoters must produce the downstream protein with a synthesis rate within a limited range. For the circuits presented in this paper, this range hardly meets the requirements for real applications, as promoters characterized by too strong or too leaky activity make the systems lose the 
correct behaviour. To overcome this problem, input RBSs can be tuned in future versions of the devices, as described in $[28,29]$, to reach the synthesis rate values identified in this paper as necessary condition for the correct working. To the same aim, the RBS upstream of luxR can also be tuned to improve the performance of the NOT gate in presence of strong input promoters. Second, crosstalk between the lux and las systems may impair the whole systems behaviour. Even though its effect cannot be decreased, the actual amount of crosstalk should be measured with ad-hoc experiments on lux and las biological elements to enable more accurate analysis through the mathematical model and a better definition of the working conditions. Third, even tuning input/selection promoters synthesis rate to achieve the expected behaviour, variation in the values of other model parameters can produce unwanted phenomena. An example is the cI-Plambda dissociation constant, which causes the nonmutually exclusive input or output selection in mux and demux respectively, for some of the simulated values.

Quantitative tests have been performed on a set of constructed circuit subsystems containing biological logic gates elements: an AND gate composed by quorum sensing elements and a NOT gate, coupled with a part of the AND gate, were characterized. The performed tests validated all the input combinations of the AND gate, which gave the expected output GFP levels. Tests also allowed to measure the steady-state characteristic of a part containing a constitutively expressed luxR gene and a reporter gene under the control of Plux promoter (test part \#3). Considering Fig. 2A, this configuration is equivalent to keeping the second input of the AND (lux) gate constant, while the first input changes by providing different concentrations of 3OC6HSL, which simulates the presence of LuxI enzyme. The measured steady-state characteristics have been useful to estimate the cut-off point between high and low states of the AND gate. The RBS-luxI module, which is involved in the mux and demux inputs, was characterized by placing promoters of different strengths upstream and measuring the concentration of the LuxI enzyme product, 3OC6HSL, in all the conditions. This can be useful to determine the critical input promoter strength required to produce a sufficient amount of 3OC6HSL to activate Plux through LuxR.

The knowledge of the autoinducer production capability of RBS-luxI (test parts \#5-10), together with the induction behaviour of luxR-Plux (test part \#3), can support the reusability of the implemented biological parts in different contexts. In fact, RBS-luxI can be used as a sender of chemical signals in the form of 3OC6HSL to engineer quorum sensing networks for cell-to-cell communication systems. On the other hand, test part \#3 can be used as a receiver of such chemical signals that can produce GFP as a function of the received 3OC6HSL molecules. When properly coupled with a heat-sensitive cI repressor, such as cI857, test part \#3 can also behave as an AND gate where inputs are 3OC6HSL and heat. 
NOT gate results (test parts \#11-12) showed that, within a specific range of input values, the constructed logic gate is functional, as Plambda can be repressed by cI and its behaviour is also well-predicted by mathematical model simulations. In this case, Plambda was turned off when cI expression was driven by a very weak promoter. This confirms the unsuitability, also predicted by the mathematical model, in real applications of the constructed NOT gate because even slight basal expression of the mux or demux selector promoter may partially repress luxR production. As discussed above, optimization should be required.

Here, only mux 2:1 and demux 1:2 have been considered. It should be noted that the behaviour of a 2-channel mux for biosensing applications could be obtained by simply using different reporters rather than complicate the circuit through the addition of more complex logic and an external input selector. Analogously, the practical utility of a 2-channel demux for enzyme-based bioprocess regulation could be obtained with simply two inputs, each with a dedicated output rather than one input and a selector. However, when input number increases, the available reporters run out and an $\mathrm{N}$-channel mux can select the desired input to be conveyed in a detectable output. In this case the selector would not be a single-bit signal, but it would be composed by $\log 2(\mathrm{~N})$ bits. The bits can be considered as sub-channels that have to respond to different stimuli, whose combination can choose the desired input to be conveyed in the output channel. On the other hand, following the same design for the selector, in an N-channel demux when output number increases it is preferable to select the output by using $\log 2(\mathrm{~N})$ selection bits rather than $\mathrm{N}$ distinct inputs.

While 2-channel mux/demux devices are a necessary step along the way to multi-channel mux/demux devices, in these cases mux and demux would be more useful when they can select among more than 2 channels. However, the scale-up process is not trivial. In fact, although truth tables and Karnaugh maps can be used again to define more complex logic networks, genetic implementation requires the knowledge of additional well-characterized biological elements that can mimic logic gates. These elements need to be assembled and included in the genetic circuit, which would have to be re-programmed and re-validated.

Recently, steps towards the design of multi-population logic circuits have been performed $[8,9]$. In these pioneering works, bacterial or yeast cell populations bearing logic functions have been chemically wired by sending and receiving molecular signals, such as autoinducers [9] or pheromones [8]. In this framework, each population represents a computational unit that remains insulated from the rest of the cell populations and for this reason the same logic gates can be implemented by using the same biological elements. Even if the mentioned design principles were successful for the implementation of a number of logic functions, we think that single-cell implementations and 
modeling of complex logic functions are equally important to discover the complexity limits of a single engineered cell population in hosting a genetic logic circuit, as well as the behaviour of the used biological elements when placed together with other modules.

In conclusion, part of the implemented logic functions has been shown to work in several tests and they can be re-used in more complex artificial systems designed to process Boolean signals. Although the parts that could lead to mux and demux devices are available in the Registry, future improvement is required to tune this parts for real applications, taking into account of the mathematical model analysis of the systems.

Acknowledgements We thank Nicolò Politi for useful discussion and help during the part characterization experiments. This project was partially supported by the Italian "Ministero dell'Università e della Ricerca" through FIRB ITALBIONET and FIRB RBIP06FH7J projects.

\section{Conflict of interest statement}

The authors have declared no conflict of interest.

\section{References}

1. M. Terzer, M. Jovanovic, A. Choutko, O. Nikolayeva, A. Korn, D. Brockhoff, F. Zurcher, M. Friedmann, R. Schutz, E. Zitzler, J. Stelling, and S. Panke. Design of a biological half adder. IET Synth. Biol., 1:53-58, 1-2 2007.

2. C. A. Voigt. Genetic parts to program bacteria. Curr. Opin. Biotechnol., 17:548-557, 2006.

3. B. P. Kramer, C. Fischer, and M. Fussenegger. Biologic gates enable transcription control in mammalian cells. Biotechnol. Bioeng., 87(4):478-484, 2004

4. R. Weiss, S. Basu, S. Hooshangi, A. Kalmbach, D. Karig, R. Mehreja, and I. Netravali. Genetic circuit building blocks for cellular computation, communications, and signal processing. Natural Computing., 2(1):47-84, 2003.

5. S. Subramanian, I. Lemischka, and R. Weiss. Engineering a 1:2 bio-multiplexer for controlled stem cell differentiation. In Computational Systems Bioinformatics Conference, page 262, 2005.

6. J. C. Anderson, C. A. Voigt, and A. P. Arkin. Environmental signal integration by a modular AND gate. Molecular System Biology, 3:133, 2007.

7. M. N. Win and C. D. Smolke. Higher-order cellular information processing with synthetic RNA devices. Science, 322:456-460, 2008.

8. S. Regot, J. Macia, N. Conde, K. Furukawa, J. Kjellen, T. Peeters, S. Hohmann, E. de Nadal, F. Posas, and R. Solé. Distributed biological computation with multicellular engineered networks. Nature, 469:207-211, 2011.

9. A. Tamsir, J. J. Tabor, and C. A. Voigt. Robust multicellular computing using genetically encoded NOR gates and chemical wires. Nature, 469:212-215, 2011. 
10. E. Andrianantoandro, S. Basu, D. Karig, and R. Weiss. Synthetic biology: new engineering rules for an emerging discipline. Molecular System Biology, 2:28, 2006

11. B. M. Frezza, S. L. Cockroft, and M. R. Ghadiri. Modular multi-level circuits from immobilized DNA-based logic gates. J. Am. Chem. Soc., 129(48):14875-9, 2007

12. C. Tan, H. Song, J. Niemi, and L. You. A synthetic biology challenge: making cells compute. Mol. BioSyst., 3:343-353, 2007.

13. M. Morris Mano and C. R. Kime. Logic and Computer Design Fundamentals. Prentice Hall, 2007.

14. W. Stallings. Computer Organization and Architecture: Designing for Performance. Prentice Hall, 2003.

15. A. M. Stevens and E. P. Greenberg. Quorum sensing in Vibrio fischeri: Essential elements for activation of the luminescence genes. Journal of Bacteriology, 179(2):557-562, 1997.

16. E. C. Pesci, J. C. Pearson, P. C. Seed, and B. H. Iglewski. Regulation of las and rhl quorum sensing in Pseudomonas aeruginosa. Journal of Bacteriology, 179(10):3127-3132, 1997.

17. M. Heinemann and S. Panke. Synthetic biology-putting engineering into biology. Bioinformatics, 22(22):2790-2799, 2006.

18. M. L. Urbanowski, C. P. Lostroh, and E. P. Greenberg. Reversible acyl-homoserine lactone binding to purified Vibrio fischeri LuxR protein. Journal of Bacteriology, 186(3):631-637, 2004

19. B.L. Hanzelka, A. M. Stevens, M.R. Parsek, T. J. Crone, and E. P. Greenberg. Mutational analysis of the Vibrio fischeri LuxI polypeptide: Critical regions of an autoinducer synthase. Journal of Bacteriology, 179(15):4882-4887, 1997.

20. M. Fussenegger. Synthetic biology: Synchronized bacterial clocks. Nature, 463:301-302, 2010.

21. K. Brenner, D. K. Karig, R. Weiss, and F. H. Arnold. Engineered bidirectional communication mediates a consensus in a microbial biofilm consortium. PNAS, 104(44):17300-17304, 2007

22. A.M. Albus, E. C. Pesci, L. J. Runyen-Janecky, S. E. West, and B. H. Iglewski. Vfr controls quorum sensing in Pseudomonas aeruginosa. Journal of Bacteriology, 179(12):3928-3935, 1997.

23. F. K. Balagaddè, H. Song, J. Ozaki, C. H. Collins, M. Barnet, F. H. Arnold, S. R. Quake, and L. You. A synthetic Escherichia coli predator-prey ecosystem. Molecular System Biology, 187:4, 2008.

24. M. Ptashne. A Genetic Switch: Phage Lambda Revisited. Cold Spring Harbor Laboratory Press, 2004.

25. M.B. Elowitz and S. Leibler. A synthetic oscillatory network of transcriptional regulators. Nature, 403:335-338, 2000.

26. B. Canton, A. Labno, and D. Endy. Refinement and standardization of synthetic biological parts and devices. Nature Biotechnology, 26(7):787-793, 2008.

27. T. F. Knight. Idempotent vector design for standard assembly of BioBricks. Technical report, DSpace, 2003. http://hdl.handle.net/1721.1/21168.

28. J. R. Kelly. Tools and reference standards supporting the engineering and evolution of synthetic biological systems. PhD thesis, Department of Biological Engineering, MIT, 2008. doi:1721.1/44917.

29. H. M. Salis, E. A. Mirsky, and C. A. Voigt. Automated design of synthetic ribosome binding sites to control protein expression. Nature Biotechnology, 27:946-950, 2009. doi:10.1038/nbt.1568. 